\title{
AN EXTENSION OF CARLSON'S THEOREM FOR ENTIRE FUNCTIONS OF EXPONENTIAL TYPE
}

BY

\author{
R. GERVAIS AND Q. I. RAHMAN( $\left.{ }^{(}\right)$
}

\begin{abstract}
The paper contains some new extensions of the well-known
\end{abstract} theorem of F. Carlson for entire functions of exponential type.

1. Introduction and statement of results. According to a well-known theorem of F. Carlson [5], [4, p. 153], if $f(z)$ is an entire function of exponential type $\tau<\pi$, and $f(n)=0$ for $n=0, \pm 1, \pm 2, \ldots$, then $f(z) \equiv 0$. This result has been extended and generalized in various ways ([4, Chapters 9, 10], [6], etc.). For example, if $f(z)$ is an entire function of exponential type $<k \pi$ where $k$ is a positive integer, and $f(z), f^{\prime}(z), \ldots, f^{(k-1)}(z)$ all vanish for $z=0, \pm 1, \pm 2, \ldots$, then $f(z) \equiv 0$. In particular, an entire function of exponential type $<2 \pi$ is completely determined by its values and those of its derivative at the integers.

While trying to extend the theory of $(0,2)$-interpolation ([7], [1]-[3]) by polynomials in points on $[-1,1]$ to $(0,2)$-interpolation by entire functions of exponential type in points on the real axis, we wanted to know to what extent an entire function of exponential type $<2 \pi$ is determined by its values and those of its second derivative at the integers, or more generally, by its values and those of its $k$ th derivative at the integers. The answer turns out to be the following.

THEOREM 1. If $f(z)$ is an entire function of exponential type $\tau<2 \pi$ such that

$$
f(n)=f^{\prime \prime}(n)=0, \quad n=0, \pm 1, \pm 2, \ldots,
$$

then $f(z)=c \sin (\pi z)$ where $c$ is a constant. Here $\tau=2 \pi$ is inadmissible.

For even $k \geqslant 4$, we have

THEOREM 2. Let $k$ be an even integer $\geqslant 4$. If $f(z)$ is an entire function of exponential type $\tau<\pi \sec (\pi / k)$ such that

$$
f(n)=f^{(k)}(n)=0, \quad n=0, \pm 1, \pm 2, \ldots,
$$

Received by the editors June 28, 1976.

AMS (MOS) subject classifications (1970). Primary 30A64, 30A66.

Key words and phrases. Entire functions of exponential type, Carlson's theorem, indicator function and indicator diagram, Bernstein's theorem.

(') This work was supported by National Research Council of Canada Grant A-3081. 
then $f(z)=c \sin (\pi z)$ where $c$ is a constant. Here $\tau$ cannot be allowed to be equal to $\pi \sec (\pi / k)$.

On the other hand, for odd $k>3$, we have

TheOREM 3. Let $k$ be an odd integer $>3$. If $f(z)$ is an entire function of exponential type $\tau<\pi \sec (\pi / 2 k)$ such that

$$
f(n)=f^{(k)}(n)=0, \quad n=0, \pm 1, \pm 2, \ldots,
$$

then $f(z) \equiv 0$. Here $\tau=\pi \sec (\pi / 2 k)$ is inadmissible.

We also prove

THEOREM 4. Let $k$ be an integer $\geqslant 2$, and $\lambda$ an arbitrary number in $[0,1)$. If $f(z)$ is an entire function of exponential type $2 \pi$ such that

(i) $|f(x)| \leqslant A+B|x|^{\lambda}$ for all real $x$ and certain constants $A, B$,

(ii) $f(n)=f^{(k)}(n)=0, n=0, \pm 1, \pm 2, \ldots$, then

$$
f(z)= \begin{cases}c_{1} \sin (\pi z)+c_{2} \sin (2 \pi z) & \text { if } k \text { is even, } \\ c \sin ^{2}(\pi z) & \text { if } k \text { is odd, }\end{cases}
$$

where $c_{1}, c_{2}$ and $c$ are constants. Here $\lambda$ cannot be allowed to be equal to 1 .

2. Lemmas. The following result will be used a number of times.

LEMMA 1. If $f(z)$ is an entire function of exponential type vanishing at the set of integers, then

$$
f(z)=\phi(z) \sin (\pi z)
$$

where $\phi(z)$ is an entire function of exponential type.

Proof. Let $f(z)$ be of exponential type $\tau$, i.e. for every $\varepsilon>0$ there exists a constant $K(\varepsilon)$ such that

$$
|f(z)|<K(\varepsilon) \exp \{(\tau+\varepsilon)|z|\}
$$

for all $z$. It is easily seen that $|\sin (\pi z)| \geqslant 1$ on the square $C_{n}$ with corners at the points $\pm\left(n+\frac{1}{2}\right) \pm i\left(n+\frac{1}{2}\right)$, where $n$ is a positive integer. Hence on $C_{n}$,

$$
|\phi(z)|<|f(z)|<K(\varepsilon) \exp \{(\tau+\varepsilon)|z|\} \leqslant K(\varepsilon) \exp \left\{(\tau+\varepsilon) \sqrt{2}\left(n+\frac{1}{2}\right)\right\} .
$$

By the maximum modulus principle the same estimate holds inside $C_{n}$. In particular, we see that the inequality

$$
|\phi(z)|<K(\varepsilon) \exp \{(\tau+\varepsilon) \sqrt{2}|z|\}
$$

holds on $|z|=n+\frac{1}{2}$. Thus, if $n-\frac{1}{2} \leqslant|z| \leqslant n+\frac{1}{2}$, then 


$$
\begin{aligned}
|\phi(z)| & \leqslant \max _{|z|=n+1 / 2}|\phi(z)|<K(\varepsilon) \exp \left\{(\tau+\varepsilon) \sqrt{2}\left(n+\frac{1}{2}\right)\right\} \\
& \leqslant K(\varepsilon) \exp \left\{(\tau+\varepsilon) \sqrt{2}|z|\left[\frac{n+\frac{1}{2}}{n-\frac{1}{2}}\right]\right\} \\
& \leqslant K(\varepsilon)\{(\tau+\varepsilon) 3 \sqrt{2}|z|\},
\end{aligned}
$$

which shows that $\phi(z)$ is of exponential type.

For the procf of Theorem 4 we shall need the following extension of Bernstein's inequality.

LEMMA 2. Let $f(z)$ be an entire function of exponential type $\tau$ such that for some $\lambda \in[0,1)$,

$$
|f(x)| \leqslant A_{1}+B_{1}|x|^{\lambda}, \quad-\infty<x<\infty,
$$

where $A_{1}, B_{1}$ are constants. Then

$$
\left|f^{\prime}(x)\right| \leqslant A^{\prime}+B^{\prime}|x|^{\lambda}, \quad-\infty<x<\infty,
$$

for some constants $A^{\prime}, B^{\prime}$ depending on $A_{1}, B_{1}, \lambda$ and $\tau$.

Proof. In order to prove Lemma 2 we may apply to

$$
g_{\delta}(z)=f(z)\{(\sin (\delta z)) /(\delta z)\}^{2}, \quad \delta>0,
$$

the identity $[4$, p. 211]

$$
F^{\prime}(x)=4 T \pi^{-2} \sum_{k=-\infty}^{\infty} \frac{(-1)^{k}}{(2 k+1)^{2}} F\left(x+\frac{2 k+1}{2 T} \pi\right),
$$

which is valid for all entire functions of exponential type $T$ belonging to $L^{2}$ on the real axis. Thus

$$
\begin{aligned}
&\left|g_{\delta}^{\prime}(x)\right| \leqslant 4(\tau+2 \delta) \pi^{-2} \sum_{k=-\infty}^{\infty} \frac{1}{(2 k+1)^{2}}\left\{A_{1}+B_{1}\left|x+\frac{2 k+1}{2(\tau+2 \delta)} \pi\right|^{\lambda}\right\} \\
&<4(\tau+2 \delta) \pi^{-2} \sum_{k=-\infty}^{\infty} \frac{1}{(2 k+1)^{2}}\left\{A_{1}+B_{1}\left|\frac{2 k+1}{2(\tau+2 \delta)} \pi\right|^{\lambda}+B_{1}|x|^{\lambda}\right\} \\
&<(\tau+2 \delta) A^{\prime \prime}+(\tau+2 \delta)^{1-\lambda} A^{\prime \prime \prime}+(\tau+2 \delta) B^{\prime \prime}|x|^{\lambda}
\end{aligned}
$$

where $A^{\prime \prime}, A^{\prime \prime \prime}, B^{\prime \prime}$ are constants depending on $A_{1}, B_{1}, \lambda$ and $\tau$. Since $g_{\delta}^{\prime}(z) \rightarrow f^{\prime}(z)$ as $\delta \rightarrow 0$, (2) follows.

For the proof of Theorem 4 we shall also need

LEMMA 3. Let $f(z)$ be an entire function of exponential type $\tau$ such that $f(n)=0$ for $n=0, \pm 1, \pm 2, \ldots$, and for some $\lambda \in[0,1)$, inequality (1) holds. 
Then $\phi(z)=f(z) / \sin (\pi z)$ is an entire function of exponential type such that

$$
|\phi(x)|<A_{2}+B_{2}|x|^{\lambda}, \quad-\infty<x<\infty,
$$

for some constants $A_{2}, B_{2}$ depending on $A_{1}, B_{1}, \lambda$ and $\tau$.

Proof. It follows from Lemma 2 that if $f(z)$ is an entire function of exponential type $\tau$ such that $f(n)=0$ for $n=0, \pm 1, \pm 2, \ldots$, and for some $\lambda \in[0,1)$ inequality (1) holds, then for certain constants $A^{*}, B^{*}$ depending on $A_{1}, B_{1}, \lambda$ and $\tau$,

$$
|f(x)|=|f(x)-f(n)| \leqslant\left(A^{*}+B^{*}|x|^{\lambda}\right)|x-n|,
$$

if $n-\frac{1}{4}<x<n+\frac{1}{4}$. On the other hand, if $n-\frac{1}{4}<x<n+\frac{1}{4}$, then

$$
|\sin (\pi x)|=\left|\int_{n}^{x} \frac{\cos (\pi t)}{\pi} d t\right| \geqslant \frac{1}{\pi \sqrt{2}}|x-n| \text {. }
$$

Hence, for $x \in\left[n-\frac{1}{4}, n+\frac{1}{4}\right]$, we have

$$
|\phi(x)|=\left|\frac{f(x)}{\sin (\pi x)}\right| \leqslant\left(A^{*}+B^{*}|x|^{\lambda}\right) \pi \sqrt{2} .
$$

If $x$ does not belong to any of the intervals $\left[n-\frac{1}{4}, n+\frac{1}{4}\right]$, then $|\sin (\pi x)|>$ $1 / \sqrt{2}$, and

$$
|\phi(x)|<\left(A_{1}+B_{1}|x|^{\lambda}\right) \sqrt{2} .
$$

The desired result follows from (3) and (4).

Besides, we shall need several other properties of entire functions of exponential type. We expect the reader to be familiar with the material contained in $\$ 5.1-\$ 5.4$ of [4]. In addition, we will use the following four lemmas.

LEMMA 4. If $H(z)$ is an entire function of exponential type then $H_{1}(z)=$ $H(z) \sin (\pi z)$ and $H_{2}(z)=H(z) \cos (\pi z)$ are both of order 1 and same type.

Proof. If we denote by $\tau(f)$ the type of an entire function $f$ and by

$$
h_{g}(\theta)=\limsup _{r \rightarrow \infty} \frac{\log \left|g\left(r e^{i \theta}\right)\right|}{r}
$$

the indicator function of an entire function $g(z)$ of exponential type, then

$$
\begin{aligned}
\tau\left(H_{1}\right) & =\max _{0<\theta<2 \pi} h_{H_{1}}(\theta)=\max _{0<\theta<2 \pi}\left\{h_{H}(\theta)+h_{\sin (\pi z)}(\theta)\right\} \\
& =\max _{0<\theta<2 \pi}\left\{h_{H}(\theta)+\pi|\sin \theta|\right\}=\max _{0<\theta<2 \pi}\left\{h_{H}(\theta)+h_{\cos (\pi z)}(\theta)\right\} \\
& =\max _{0<\theta<2 \pi} h_{H_{2}}(\theta)=\tau\left(H_{2}\right) .
\end{aligned}
$$

LEMMA 5. If $G(z)$ is an entire function of order 1 type $>\pi$, then $g(z)=$ $G(z) \sin (\pi z)$ is of order 1 type $>\pi \sqrt{2}$. 
Proof. According to a well-known property of the indicator function, if $0<\theta_{2}-\theta_{1}<\pi, h_{G}\left(\theta_{1}\right)<h_{1}, h_{G}\left(\theta_{2}\right)<h_{2}$, then $[4$, p. 66]

$$
h_{G}(\theta)<\frac{h_{1} \sin \left(\theta_{2}-\theta\right)+h_{2} \sin \left(\theta-\theta_{1}\right)}{\sin \left(\theta_{2}-\theta_{1}\right)}, \quad \theta_{1}<\theta<\theta_{2} .
$$

Hence, if $h_{G}(\pi / 4), h_{G}(3 \pi / 4), h_{G}(5 \pi / 4), h_{G}(7 \pi / 4)$ were all less than $\pi / \sqrt{2}$ then $h_{G}(\theta)$ would be less than $\pi$ for all $\theta$ contradicting the fact that $G(z)$ is of type $>\pi$. Thus

$$
\max \left\{h_{G}(\pi / 4), h_{G}(3 \pi / 4), h_{G}(5 \pi / 4), h_{G}(7 \pi / 4)\right\}>\pi / \sqrt{2},
$$

and

$$
\begin{aligned}
\tau(g) & =\max _{\theta} h_{g}(\theta)>\max \left\{h_{g}(\pi / 4), h_{g}(3 \pi / 4), h_{g}(5 \pi / 4), h_{g}(7 \pi / 4)\right\} \\
& >\pi / \sqrt{2}+\pi / \sqrt{2}=\pi \sqrt{2} .
\end{aligned}
$$

LEMMA $6[4$, p. 156]. If $f(z)$ is an entire function of exponential type whose indicator diagram has width at most $2 \pi$ in the direction of the imaginary axis, and does not contain two horizontal straight line segments whose perpendicular distance apart is $2 \pi$, then if $f(z)=0$ for $z=0, \pm 1, \pm 2, \ldots$,

$$
f(z)=e^{\alpha z} \phi(z) \sin (\pi z),
$$

where $\phi(z)$ is an entire function of zero exponential type.

LEMMA 7 [4, p. 83]. If $f(z)$ is an entire function of exponential type, $h_{f}( \pm \pi / 2)<0$, and $f(x)=O\left(|x|^{p}\right), x \rightarrow \pm \infty$, then $f(z)$ is a polynomial of degree not exceding $p$.

\section{Proofs of theorems.}

Proof OF THEOREM 1. Let $f(z)$ satisfy the hypotheses of Theorem 1. Since $f(z)$ vanishes at the set of integers it follows from Lemma 1 that $f(z)=\phi(z)$ - $\sin (\pi z)$ where $\phi(z)$ is an entire function of exponential type. Thus

$$
f^{\prime \prime}(z)=\left\{-\pi^{2} \phi(z)+\phi^{\prime \prime}(z)\right\} \sin (\pi z)+2 \pi \phi^{\prime}(z) \cos (\pi z) .
$$

By hypothesis $f^{\prime \prime}(z)$ vanishes for $z=0, \pm 1, \pm 2, \ldots$ Therefore $\phi^{\prime}(z)$ should vanish for $z=0, \pm 1, \pm 2, \ldots$. Again, by Lemma 1 ,

$$
\phi^{\prime}(z)=\psi(z) \sin (\pi z)
$$

where $\psi(z)$ is an entire function of exponential type. The function $\psi(z)$ must be identically zero. For if $\psi(z) \neq 0$, then by [4, Theorem 5.4.4, p. 76]

$$
h_{\psi}(\pi / 2)+h_{\psi}(-\pi / 2) \geqslant 0
$$

where

$$
h_{\psi}(\theta)=\limsup _{r \rightarrow \infty} \frac{\log \left|\psi\left(r e^{i \theta}\right)\right|}{r}
$$


is the indicator function of $\psi(z)$. From (5) and (6) it would follow that

$$
\max \left\{h_{\phi^{\prime}}(\pi / 2), h_{\phi^{\prime}}(-\pi / 2)\right\} \geqslant \pi,
$$

and so if $w(z)=\phi^{\prime}(z) \sin (\pi z)$, then

$$
\tau(w) \geqslant \max \left\{h_{w}(\pi / 2), h_{w}(-\pi / 2)\right\} \geqslant \pi+\pi=2 \pi .
$$

Since by Lemma 4,

$$
\text { type of } \phi(z) \cos (\pi z)=\text { type of } \phi(z) \sin (\pi z)=\tau(f)<2 \pi \text {, }
$$

this would imply that $f^{\prime}(z)$ is of order 1 type $\geqslant 2 \pi$, which is a contradiction since $f(z)$ is supposed to be of exponential type $<2 \pi$. Thus we have shown that $\psi(z) \equiv 0$ and hence $\phi(z)$ is a constant. This completes the proof of Theorem 1.

The example $f(z)=\sin (2 \pi z)$ shows that $\tau=2 \pi$ is inadmissible.

Proof of Theorems 2 AND 3. By Lemma $1, f(z)=\phi(z) \sin (\pi z)$ where $\phi(z)$ is an entire function of exponential type. Hence

$$
\begin{aligned}
f^{(k)}(z)= & \sum_{j=0}^{k}\left(\begin{array}{l}
k \\
j
\end{array}\right) \phi^{(k-j)}(z) \frac{d^{j}}{d z^{j}}\{\sin (\pi z)\} \\
= & \left\{\sum_{\mu=0}^{[k / 2]}(-1)^{\mu} \pi^{2 \mu}\left(\begin{array}{c}
k \\
2 \mu
\end{array}\right) \phi^{(k-2 \mu)}(z)\right\} \sin (\pi z) \\
& +\left\{\sum_{\nu=1}^{[(k+1) / 2]}(-1)^{\nu-1} \pi^{2 \nu-1}\left(\begin{array}{c}
k \\
2 \nu-1
\end{array}\right) \phi^{(k-2 \nu+1)}(z)\right\} \cos (\pi z) .
\end{aligned}
$$

From the fact that $f^{(k)}(z)$ vanishes at the set of integers it follows that the function

$$
G(z)=\sum_{\nu=1}^{[(k+1) / 2]}(-1)^{\nu-1} \pi^{2 \nu-1}\left(\begin{array}{c}
k \\
2 \nu-1
\end{array}\right) \phi^{(k-2 \nu+1)}(z)
$$

also vanishes at the set of integers, and hence by Lemma 1 ,

$$
\text { (7) } G(z)=\sum_{\nu=1}^{[(k+1) / 2]}(-1)^{\nu-1} \pi^{2 \nu-1}\left(\begin{array}{c}
k \\
2 \nu-1
\end{array}\right) \phi^{(k-2 v+1)}(z)=\psi(z) \sin (\pi z) \text {. }
$$

where $\psi(z)$ is an entire function of exponential type. Here $\psi(z)$ must be identically zero. Suppose, if possible, that $\psi(z) \neq 0$. From (6) we conclude that $\phi(z)$ is of order 1 type $\geqslant \pi$, and so by Lemma $5, f(z)$ is of order 1 type $>\pi \sqrt{2}$ which is a contradiction since $f(z)$ is assumed to be of exponential type $<\pi \sec (\pi / k)$ if $k$ is an even integer $>4$ and of exponential type $<$ $\pi \sec (\pi / 2 k)$ if $k$ is an odd integer $\geqslant 3$. Hence $\psi(z) \equiv 0$. But then $\phi(z)$ being a solution of the differential equation 


$$
\sum_{\nu=1}^{[(k+1) / 2]}(-1)^{\nu} \pi^{2 \nu-1}\left(\begin{array}{c}
k \\
2 \nu-1
\end{array}\right) \phi^{(k-2 \nu+1)}(z)=0
$$

must be of the form

$$
\phi(z)=\sum_{\nu=1}^{k-1} c_{\nu} \exp \left(\pi z \cot \frac{\nu \pi}{k}\right)
$$

where $c_{1}, c_{2}, \ldots, c_{k-1}$ are constants. If $k$ is even then all the constants $c_{1}$, $c_{2}, \ldots, c_{k-1}$ except $c_{k / 2}$ should be zero for otherwise $\phi(z)$ will be of order 1 type $\geqslant \pi \tan (\pi / k)$ which will mean that $f(z)$ is of order 1 type $>$ $\pi \sec (\pi / k)$. Hence $\phi(z)$ is a constant and $f(z)$ is of the form $c \sin (\pi z)$. If $k$ is odd then all the constants $c_{1}, c_{2}, \ldots, c_{k-1}$ in (8) should be zero or else $\phi(z)$ will be of order 1 type $\geqslant \pi \tan (\pi / 2 k)$. This would imply that $f(z)$ is of order 1 type $\geqslant \pi \sec (\pi / 2 k)$. Hence $\phi(z)$ is identically zero and so is $f(z)$. With this Theorems 2 and 3 are completely proved.

The example $f(z)=\exp (z \pi \tan (\pi / k)) \sin (\pi z)$ shows that in Theorem 2 $\tau=\pi \sec (\pi / k)$ is inadmissible. On the other hand, the example

$$
f(z)=\exp (z \pi \tan (\pi / 2 k)) \sin (\pi z)
$$

shows that $\tau=\pi \sec (\pi / 2 k)$ is inadmissible in Theorem 3.

Proof of Theorem. 4. By Lemma $3, f(z)=\phi(z) \sin (\pi z)$ where $\phi(z)$ is an entire function of exponential type such that

$$
|\phi(x)| \leqslant A_{2}+B_{2}|x|^{\lambda}, \quad-\infty<x<\infty,
$$

for some constants $A_{2}$ and $B_{2}$. In view of (9) we can even claim that $\phi(z)$ is of exponential type $\pi$. As in the proofs of Theorems 2 and 3 it follows that the function

$$
G(z)=\sum_{\nu=1}^{[(k+1 / 2)]}(-1)^{\nu-1} \pi^{2 \nu-1}\left(\begin{array}{c}
k \\
2 \nu-1
\end{array}\right) \phi^{(k-2 \nu+1)}(z)
$$

vanishes at the set of integers. Besides $G(z)$ is of exponential type $\pi$ and by Lemma 2,

$$
|G(x)| \leqslant A_{3}+B_{3}|x|^{\lambda}, \quad-\infty<x<\infty,
$$

for some constants $A_{3}$ and $B_{3}$ so that $G(z)$ satisfies the hypotheses of Lemma 6. Consequently,

$$
G(z)=e^{\alpha z} \omega(z) \sin (\pi z)
$$

where $\alpha$ is real and $\omega(z)$ is an entire function of zero exponential type. Because of (10), $\alpha$ should be zero, and $h_{\omega}( \pm \pi / 2)<0$. Thus using Lemma 7 we conclude that $G(z)=\beta \sin (\pi z)$ where $\beta$ is a constant. In other words, $\phi(z)$ is a solution of the differential equation 


$$
\sum_{\nu=1}^{[(k+1) / 2]}(-1)^{\nu-1} \pi^{2 \nu-1}\left(\begin{array}{c}
k \\
2 \nu-1
\end{array}\right) \phi^{(k-2 \nu+1)}(z)=\beta \sin (\pi z) .
$$

According as $k$ is even or odd the most general solution of (12) is

$$
\phi(z)=\sum_{\nu=1}^{k-1} c_{\nu} \exp \left(\pi z \cot \frac{\nu \pi}{k}\right)+(-1)^{k / 2} \frac{\beta}{\pi^{k} \sum_{j=1}^{k / 2}\left(\begin{array}{l}
k j-1 \\
2 j
\end{array}\right)} \cos (\pi z)
$$

or

(14) $\phi(z)=\sum_{\nu=1}^{k-1} c_{\nu} \exp \left(\pi z \cot \frac{\nu \pi}{k}\right)+(-1)^{(k+1) / 2} \frac{\beta}{\pi^{k} \sum_{j=1}^{(k+1) / 2}(2 j-1)} \sin (\pi z)$,

respectively, where $c_{1}, c_{2}, \ldots, c_{k-1}$ are constants. In view of (9), all these constants, except $c_{k / 2}$ when $k$ is even, should be zero. Hence

$$
\phi(z)=c_{k / 2}+(-1)^{k / 2} \frac{\beta}{\pi^{k} \sum_{j=1}^{k / 2}(2 j-1)} \cos (\pi z),
$$

or

$$
\phi(z)=(-1)^{(k+1) / 2} \frac{\beta}{\pi^{k} \sum_{j=1}^{(k+1) / 2}\left(2_{2 j-1}^{k}\right)} \sin (\pi z),
$$

according as $k$ is even or odd, respectively. From this the desired result follows.

According as $k$ is even or odd the functions

$$
\frac{\sin ^{2}(\pi z)}{\pi}-\frac{z}{k} \sin (2 \pi z) \text { and } \frac{\sin (2 \pi z)}{2 \pi}+\frac{2 z}{k} \sin ^{2}(\pi z),
$$

respectively, show that in Theorem $4 \lambda$ cannot be allowed to be equal to 1 .

\section{REFERENCES}

1. J. Balázs and P. Turán, Notes on interpolation. II: Explicit formulae, Acta Math. Acad. Sci. Hungar. 8 (1957), 201-215. MR 19, 544.

2. Notes on interpolation. III: Convergence, Acta Math. Acad. Sci. Hungar. 9 (1958), 195-214. MR 20 \# 4725.

3. $\longrightarrow$ Notes on interpolation. IV: Inequalities, Acta. Math. Acad. Sci. Hungar. 9 (1958), 243-258. MR 21 \#2135.

4. R. P. Boas, Jr., Entire functions, Academic Press, New York, 1954. MR 16, 914.

5. F. Carlson, Sur une classe de séries de Taylor, Thesis, Upsala, 1914.

6. Q. I. Rahman, Interpolation of entire functions, Amer. J. Math. 87 (1965), 1029-1076. MR 32 \#7746.

7. J. Surányi and P. Turán, Notes on interpolation. I: On some interpolatorical properties of the ultraspherical polynomials, Acta Math. Acad. Sci. Hungar. 6 (1955), 67-80. MR 17, 148.

Département de Mathématiques, Universtté de Montréal, Montréal, Québec, Canada 